\title{
Implementation of the LOOP pathway-a framework for the management of ocular tuberculosis across the United Kingdom?
}

\author{
Huzaifa Malick ${ }^{1} \cdot$ Martin Wiselka $^{1} \cdot$ Periyasamy Kumar $^{1} \cdot$ Bharat Kapoor $^{1}$
}

Received: 15 October 2020 / Revised: 16 November 2020 / Accepted: 26 November 2020 / Published online: 14 January 2021

(c) The Author(s), under exclusive licence to The Royal College of Ophthalmologists 2021

The number of people in England with tuberculosis (TB) has fallen from a peak of 8280 in 2011 to 4655 in 2018 representing a $44 \%$ reduction [1]. Although this is very encouraging, TB still presents a very real challenge to health services in both developed and undeveloped countries, especially when TB affects the eye.

Ocular TB has always been a particularly difficult diagnosis to make for several reasons. The disease can present in many different ways in the eye and presumed disease is often not possible to confirm on testing, heightening the difficulty in diagnosis, management and followup. A missed diagnosis can lead to long-term visual morbidity and even mortality, yet an incorrect diagnosis can lead to prolonged, unnecessary treatment with long-term side effects. The pan-LOndon Ocular tuberculosis Pathway (LOOP) published by Petrushkin et al. [2] aims to streamline this process for patients presenting to TB services in London. Patients with granulomatous uveitis were divided into those with and those without an ocular phenotype consistent with TB. Interferon-gamma release assay (IGRA) testing was offered to both subsets of patients and those whom were positive, regardless of ocular phenotype, were referred for systemic evaluation by TB services. A negative IGRA test still resulted in referral to TB services for those with an ocular phenotype for TB, but not for those without. IGRA testing is becoming more commonplace in diagnostic algorithms, but is not without significant caveats. Ang et al. found that the accuracy of diagnosing tuberculosisassociated uveitis (TAU) increased significantly when both the tuberculin skin test (TST) and TSPOT $^{\circledR}$ tests were used in conjunction. Patients with both tests positive were 2.16 (95\% CI, 1.23-3.80) times more likely to have TAU

Bharat Kapoor

Bharat.Kapoor@uhl-tr.nhs.uk

1 Leicester Royal Infirmary, Infirmary Square, Leicester LE1 5WW, UK
[3] although it should be noted that this study was conducted in Singapore, with a significantly increased burden of TB compared to western Europe [4].

Whom to test is just as difficult a question as how to test. The United Kingdom (UK) National Institute for Clinical Excellence (NICE) guidelines from 2016 recommend IGRA testing in patients with positive TST where active TB has been ruled out and in all immunocompromised patients [5]. Other health agencies suggest avoiding any testing at all in low-risk patients, with the Centers for Disease Control and Prevention in the United States (US), for example, recommending replacing TST with IGRA in high-risk groups only and avoiding any testing in low-risk groups (https://www. cdc.gov/. Accessed Sept 2020). Due to low pre-test probability in cases of low clinical suspicion, up to $90 \%$ of positive IGRAs can be false positives [6]. This is in keeping with Bayes' theorem, which shows that even when using a very sensitive test, the lower the base rate of a given outcome the higher the probability of a false positive result, as true positives are far outnumbered by false positives. Given this, Albini et al. recommend the use of IGRAs as a primary test only in cases of high suspicion for ocular TB, given increased pre- and post-test probability [6-8]. Another area that needs further research is interpretation of the numerical value of a positive IGRA result. One of the major limitations of an IGRA test is the lack of prospective data about predicting the risk of active TB in patients with different levels of IGRA. It must be reiterated that TB uveitis is ultimately very much a clinical diagnosis and tests can only partially inform decision making.

Pre-test probability is a crucial factor in deciding upon the weighting given to any particular test when dealing with suspected ocular TB. An estimation of the positive predictive value (PPV) of a test suggests the likelihood that disease is present with a positive test. As noted by Rosenbaum [9] in his editorial, the PPV remains low if the pre-test probability is low. Hong et al. looked at factors associated with positive screening tests for ocular TB and interestingly diagnosed ocular TB in no US-born patients in their study. 
Rosenbaum comments that an estimated PPV of 4.8 vs. $30 \%$ (with a sensitivity of $75 \%$ and specificity of $85 \%$ ) in US nationals compared to foreign-born patients, respectively, who had a positive PPD and had definite or presumed ocular TB and treated as such would likely be a justification of anti-tuberculosis therapy (ATT) for many clinicians $[9,10]$. For this to be a clinically relevant calculation, some estimation of TB prevalence needs to be factored in the patient cohort of each clinician, but this will likely prove to be challenging given the difficulty in maintaining accurate live data on TB demographics in any given area. The numbers are likely to vary significantly across the UK and are subject to constant change. A recent British Ocular Surveillance Unit study into ocular TB prevalence across the UK found marked differences in incidence across the country. London and Manchester were identified as hotspots with Pakistan, India and Africa representing the commonest source of emigrant patients with ocular TB in the UK at the time of the study, but this has likely already changed given recent world events [11]. With this in mind, it is likely that pre-test probability and thus PPV estimates need to be tailored depending on the demographics of the population in question. We feel that the creation of a UK wide treatment pathway would therefore prove to be difficult.

With regards to the contentious issue of duration of ATT therapy, authors of the LOOP pathway suggested 9 months as a balance between 6 and 12 months. NICE guidance suggests 6-month duration for therapy, which is largely in keeping with practice across the UK and is our preferred duration of therapy $[2,5,11]$. It must be pointed out that factors such as drug resistance and concomitant immunosuppression would alter the duration of therapy required, and this is likely to be a greater factor in densely populated, mixed communities such as London. Further research is required in this area.

Given the difficulty surrounding much of the decision making for patients with suspected ocular TB, extrapolation of algorithms such as the LOOP pathway into a national framework would be highly desirable. However, given the significant demographic variability of this subset of patients across the UK, it would probably be sensible for regional centres to provide local frameworks of management, with regular auditing of outcomes driving changes and updates of diagnostic and treatment pathways.

\section{Compliance with ethical standards}

Conflict of interest The authors declare that they have no conflict of interest.

Publisher's note Springer Nature remains neutral with regard to jurisdictional claims in published maps and institutional affiliations.

\section{References}

1. UK Government/Public Health England. Tuberculosis in England-annual report 2019. https://www.gov.uk/government/ publications. Accessed Sept 2020.

2. Petrushkin H, Sethi C, Potter J, Martin L, Russell G, White V, et al. Developing a pathway for the diagnosis and management of ocular tuberculosis. The pan-LOndon Ocular tuberculosis Pathway-LOOP. Eye. 2020;34:805-8. https://doi.org/10.1038/ s41433-019-0543-7.

3. Ang M, Wong W, Ngan CC, Chee SP. Interferon-gamma release assay as a diagnostic test for tuberculosis-associated uveitis. Eye. 2012;26:658-65. https://doi.org/10.1038/eye.2012.1.

4. UK Government/Public Health England. Tuberculosis by country: rates per 100,000 people. https://www.gov.uk/government/publica tions. Accessed Sept 2020.

5. NICE (National Institute of Clinical Excellence). NICE guidelines -tuberculosis. https://www.nice.org.uk/guidance/ng33. Accessed Sept 2020.

6. Albini TA, Karakousis PC, Rao NA. Interferon-gamma release assays in the diagnosis of tuberculous uveitis. Am J Ophthalmol. 2008; $146: 486-8$.

7. Westbury CF. Bayes' rule for clinicians: an introduction. Front Psychol. 2010;1:192. https://doi.org/10.3389/fpsyg.2010.00192.

8. Lee C, Agrawal R, Pavesio C. Ocular tuberculosis-a clinical conundrum. Ocul Immunol Inflamm. 2016;24:237-42. https://doi. org/10.3109/09273948.2014.985387.

9. Rosenbaum JT. To be or not TB? $\mathrm{Br} \mathrm{J}$ Ophthalmol. 2014;98:999-1000. https://doi.org/10.1136/bjophthalmol-2014304883.

10. Hong BK, Khanamiri HN, Bababeygy SR, Rao NA. The utility of routine tuberculosis screening in county hospital patients with uveitis. Br J Ophthalmol. 2014;98:1091-5.

11. Shirley K, Dowlut S, Silvestri J, Pavesio C, Foot B. Presumed ocular tuberculosis in the United Kingdom: a British Ophthalmological Surveillance Unit (BOSU) study. Eye. 2020;34:1835-41. https://doi.org/10.1038/s41433-019-0748-9. 